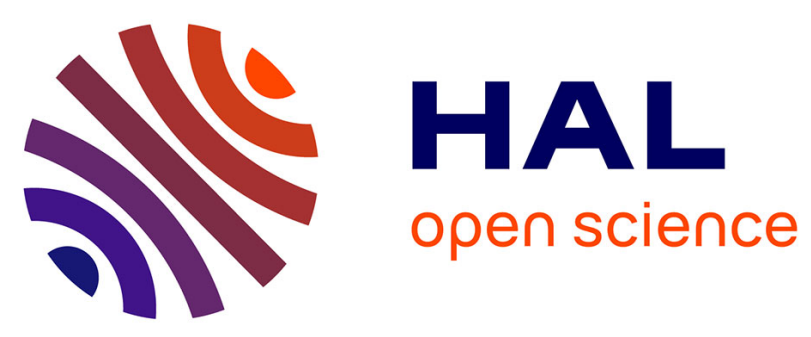

\title{
Cas locatifs et prédication
}

Richard Renault

\section{To cite this version:}

Richard Renault. Cas locatifs et prédication. Syntaxe et Sémantique, 2005, Aux marges de la prédication, 1 (6), pp.57-86. 10.3917/ss.006.0057 . hal-00012689

\section{HAL Id: hal-00012689 \\ https://hal.science/hal-00012689}

Submitted on 26 Oct 2005

HAL is a multi-disciplinary open access archive for the deposit and dissemination of scientific research documents, whether they are published or not. The documents may come from teaching and research institutions in France or abroad, or from public or private research centers.
L'archive ouverte pluridisciplinaire HAL, est destinée au dépôt et à la diffusion de documents scientifiques de niveau recherche, publiés ou non, émanant des établissements d'enseignement et de recherche français ou étrangers, des laboratoires publics ou privés. 


\title{
Cas locatifs et prédication
}

\author{
Richard Renault \\ Université de Caen Basse-Normandie, CRISCO (UMR 6170, CNRS) \\ renault@sc-homme.unicaen.fr
}

\begin{abstract}
Résumé: M'appuyant sur la typologie des systèmes casuels de Hjelmslev, je propose de rendre compte du système des cas locatifs du finnois (6 à 9 cas) par une analyse en traits morphologiques. Les deux dimensions de ce système casuel - l'orientation et la localisation - sont décrites au moyen de traits renvoyant à des têtes fonctionnelles indépendantes sur le plan syntaxique et à des entités / fonctions sur le plan sémantique, et chacune d'elles repose sur une dépendance syntaxique distincte: l'orientation relève de la rection (réduite à la sous-catégorisation) tandis que la localisation relève de la prédication syntaxique.

Summary: Basing my arguments on Hjelmslev's typology of casual systems, I will account for the system of locative cases in Finnish (there are from 6 to 9 cases) by means of morphological features. The two dimensions of this casual system - orientation and localisation - will be described with the help of features realized as independent functional heads, each one corresponding to one of the semantic entitie / function of Jackendoff's conceptual structure. The justification for this analysis relies on the distinct syntactic dependencies of these functional heads: orientation depends on head-complement relation (sub-categorization) while localisation pertains to syntactic predication.
\end{abstract}

\section{Introduction}

Chacun des six exemples finnois ${ }^{1}$ suivants présente un suffixe de cas locatif différent, là où en français deux prépositions seulement sont employées pour exprimer les mêmes relations spatiales entre le sujet et le complément locatif:
(1) a. Suvi on ravintolassa
Suvi est restaurant+INESSIF ${ }^{2}$
"Suvi est dans le restaurant"
b. Suvi menee ravintolaan
Suvi va restaurant+ILLATIF
a'. Suvi on kadulla
Suvi est rue+ADESSIF
"Suvi est dans la rue»
b'. Suvi menee kadulle
Suvi va rue+ALLATIF

1. Je remercie Meri Laajavarja pour sa relecture des exemples finnois. Ses corrections et commentaires m'ont été très utiles lors de la rédaction finale de cet article. Je remercie également Valérie Amary, Jacques François, et Isabelle Haïk pour toutes leurs remarques et suggestions durant l'élaboration de ce travail dans le cadre de l'équipe «Prédication » du CRISCO.

2. Seuls les morphèmes mentionnés dans le texte sont mis en évidence dans les exemples et identifiés dans la glose. 
«Suvi va dans le restaurant»

c. Suvi tulee ravintolasta

Suvi vient restaurant+ÉLATIF

"Suvi vient du restaurant"
"Suvi va dans la rue»

c'. Suvi tulee kadulta

Suvi vient rue+ABLATIF

"Suvi vient de la rue" ${ }^{3}$

La différence entre les deux langues concerne, d'une part, l'expression du mouvement ou de l'absence de mouvement et, d'autre part, la nature de la relation spatiale entre la cible et le site (respectivement le sujet et le locatif dans les exemples précédents). Outre le fait que le français ne fait pas de distinction entre l'absence de mouvement et le mouvement de rapprochement dans le choix de la préposition, le point important pour l'objet de cet article est que le finnois présente une opposition systématique entre les exemples de gauche et les exemples de droite. Cette opposition entre deux séries de suffixes casuels n'est ni d'ordre lexical ni d'ordre syntaxique. Elle est notamment indépendante du choix du verbe et des catégories fonctionnelles qui lui sont associées. Il ne s'agit donc pas d'un fait de rection. Cette opposition est par contre sensible au contenu lexical du locatif (un restaurant n'a pas les mêmes propriétés géométriques qu'une rue ${ }^{5}$ ) et à la nature du rapport spatial entre le sujet et le locatif (le sujet est une personne susceptible de se mouvoir dans l'espace délimité par le locatif). Comme on le verra par la suite, cette opposition d'ordre sémantique repose sur une relation de prédication syntaxique (relation sujet - prédicat).

3. Le contraste entre les deux langues peut être réduit (trois prépositions en français au lieu de deux) si l'opposition entre les exemples de gauche et les exemples de droite a une expression distincte dans les deux langues. Dans les exemples suivants, l'opposition est du type relation d'inclusion $v s$ relation de proximité / contiguïté :

a. Suvi on ravintolassa Suvi est restaurant+INESSIF "Suvi est dans le restaurant"

b. Suvi menee ravintolaan Suvi va restaurant+ILLATIF "Suvi va dans le restaurant»

c. Suvi tulee ravintolasta

Suvi vient restaurant+ÉLATIF

"Suvi vient du restaurant» a'. Suvi on asemalla Suvi est gare+ADESSIF

"Suvi est à la gare"

b'. Suvi menee asemalle

Suvi va gare+ALLATIF

"Suvi va à la gare"

c'. Suvi tulee asemalta

Suvi vient gare+ABLATIF

"Suvi vient de la gare»

4. Vandeloise 1986 : La cible est l'objet à localiser et le site l'objet de référence.

5. Une des propriétés pertinentes pour rendre compte de l'opposition entre les deux séries casuelles de (1), est la différence entre un espace fermé (le restaurant) et un espace ouvert (la rue). Dans tous les exemples de la note précédente, le complément locatif est un bâtiment (espace fermé), et cependant, l'opposition est maintenue. Mais cette fois il y a opposition entre inclusion et proximité / contiguïté. Un rapport de proximité / contiguïté peut couvrir également les cas d'inclusion dans la mesure où le site est envisagé comme repère et non comme un espace (" être à la gare » signifie aussi bien «être dans la gare " que «être devant /à côté de la gare).
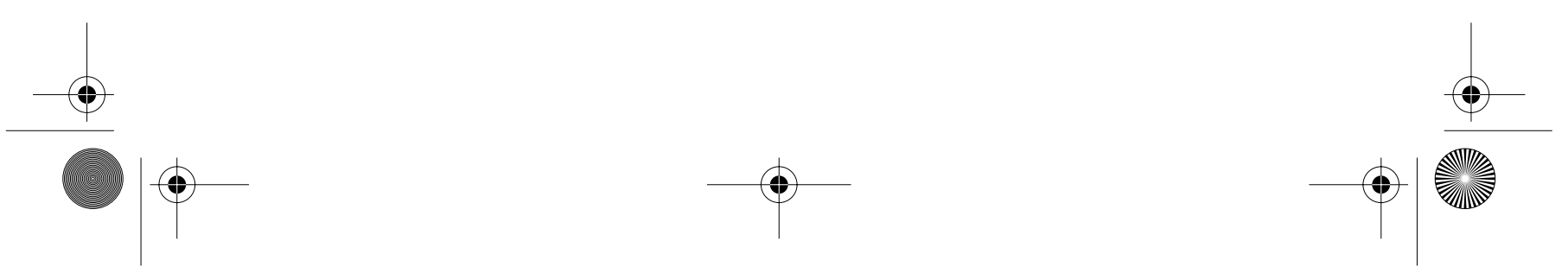
Pour rendre compte de la diversité des cas locatifs du finnois et du système casuel sous-jacent, je propose l'hypothèse suivante :

(2) La distribution des cas locatifs du finnois relèvent de la rection et de la prédication.

Dans les exemples (1a-c), la rection est représentée par la contrainte lexicale du verbe qui impose soit un cas locatif de mouvement - rapprochement (illatif, allatif) ou éloignement (élatif, ablatif) - soit un cas locatif d'absence de mouvement (inessif, adessif), tandis que la relation de prédication propre aux suffixes de cas locatifs est représentée par l'opposition entre la série casuelle de gauche (inessif, illatif, élatif) et la série de droite (adessif, allatif, ablatif).

Afin de montrer en quoi la prédication syntaxique est impliquée dans le système et le fonctionnement des cas locatifs, j’introduirai tout d'abord la typologie des systèmes casuels de Hjelmslev ${ }^{6}$, en me limitant aux cas locatifs. Cette typologie fondée sur le contenu des cas fournira l'ossature d'une description des cas locatifs du finnois sous la forme d'un système à deux dimensions dans lequel les suffixes casuels sont représentés par une matrice de traits morphologiques. Le recours à ces traits sera justifié sur le plan de l'expression et du contenu ainsi que par la sous-catégorisation des catégories régissantes. Dans la dernière partie, je montrerai comment l'unique trait locatif qui ne relève pas de la sous-catégorisation, est pris en compte au niveau syntaxique par la relation de prédication.

\section{Typologie des systèmes casuels de Hjelmslev}

Hjelmslev $^{7}$ propose une typologie des systèmes casuels fondée sur trois dimensions, et chacune de ces trois dimensions comporte au moins une opposition ${ }^{8}$. Ces trois dimensions sont 1) la direction, 2) la cohérence-incohérence, 3) la subjectivité-objectivité. La direction est représentée principalement par l'opposition entre éloignement et rapprochement, l'opposition entre cohérence et incohérence met en jeu la nature du rapport entre la cible et le site, et l'opposition entre subjectivité et objectivité introduit le point de vue du locuteur dans la relation entre la cible et le site. Selon Hjelmslev, les trois dimensions sont hiérarchisées. Ce point est schématisé par la série d'implications (3).

(3) subjectivité-objectivité $\Rightarrow$ cohérence-incohérence $\Rightarrow$ direction

Les systèmes casuels qui ne comportent qu'une seule dimension (il ne peut s'agir que de la direction, en vertu de la hiérarchie précédente)

6. Hjelmslev 1935, 1937

7. Ibid.

8. On trouvera une présentation de cette typologie dans Serbat 1981 et Blake 1994. 
sont les plus nombreux tandis que les systèmes à trois dimensions sont extrêmement rares; ils ne sont attestés que dans deux langues du Caucase (le tabassaran et le lak). Cette répartition des langues en fonction des trois dimensions est corrélée au nombre de cas; les langues qui n'ont qu'une dimension casuelle n'ont pas plus d'une dizaine de cas, les langues qui se caractérisent par un système casuel à deux dimensions ont entre dix et vingt cas, et les rares langues qui présentent les trois dimensions en ont le plus grand nombre, plus d'une trentaine de cas (voir cependant le commentaire des exemples $11 \mathrm{a}-\mathrm{b}$ ).

Le système de cas locatifs le plus simple est celui qui n'a qu'une seule dimension (la direction). Un tel système est représenté par le turc. Les trois cas locatifs sont illustrés par les exemples (5a-c) :

(4) Cas locatifs du turc (une dimension)

\begin{tabular}{|c|c|c|}
\hline \multirow{3}{*}{ 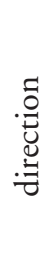 } & $\begin{array}{c}\text { locatif } \\
-d E^{9}\end{array}$ & pas de mouvement \\
\hline & $\begin{array}{c}\text { directif } \\
-(y) E\end{array}$ & rapprochement \\
\hline & $\begin{array}{c}\text { ablatif } \\
-d E n\end{array}$ & éloignement \\
\hline
\end{tabular}

(5) a. Evde kalyorum

maison+LOCATIF je reste

"Je reste à la maison"

b. Bankaya gidiyorum

banque+DIRECTIF je vais

"Je vais à la banque"

c. Evden geliyorum

maison+ABLATIF je viens

"Je viens de la maison"

Le finnois est un parfait représentant des systèmes à deux dimensions; direction et cohérence-incohérence forment un système de six cas locatifs. Comme en turc, la direction contient une opposition à trois termes: absence de mouvement (inessif et adessif), éloignement (élatif et ablatif) et rapprochement (illatif et allatif), tandis que la deuxième dimension, la cohérence-incohérence, est représentée par l'opposition entre deux séries de cas: les cas internes (inessif, élatif et illatif), et les cas externes (adessif, ablatif et allatif). Pour l'essentiel, l'opposition entre les deux séries touche à la nature du rapport spatial; rapport spatial inclusif entre

9. La majuscule représente une voyelle [ ${ }$ ARRIERE] soumise au processus d'harmonie vocalique $(\mathrm{E}=\mathrm{e}$ ou $\mathrm{a})$.
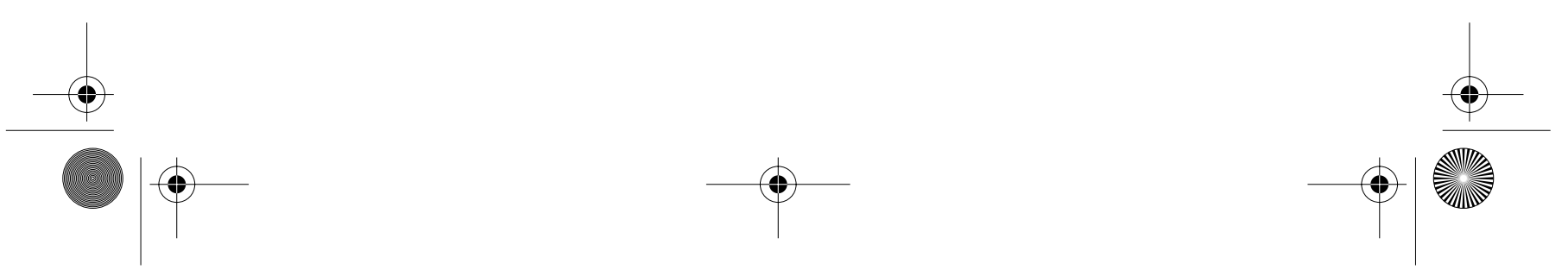
la cible et le site pour les cas internes et un rapport non inclusif pour les cas externes. Les différents cas locatifs du finnois sont illustrés en (1).

(6) Cas locatifs du finnois (deux dimensions)

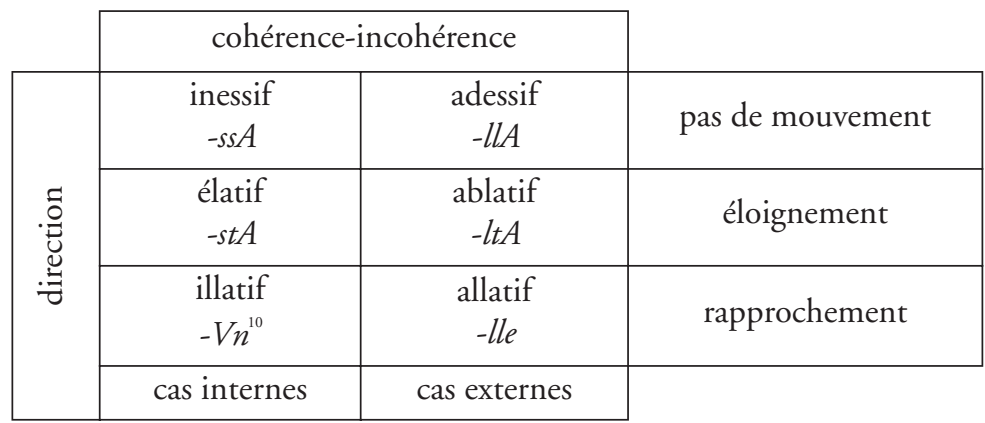

En (1a-a') le verbe statique olla "être» est accompagné d'un inessif ou d'un adessif, le rapport spatial entre la cible (le locuteur) et le site (la salle ou la rue) est de nature inclusive en (1a) et non inclusive en ( $\left.1 \mathrm{a}^{\prime}\right)$; la salle est un espace fermé, tandis que la rue représente un espace ouvert par rapport au locuteur. Ce rapport spatial est le même dans les exemples (1b-b') et (1c-c') qui contiennent cette fois un verbe de mouvement.

En plus de la direction et de la cohérence-incohérence, la troisième dimension fait intervenir une opposition entre une relation spatiale envisagée de manière objective ou de manière subjective. À priori, cette troisième dimension est censée prendre en compte les deux axes de la représentation spatiale: l'axe vertical (opposition dessus/dessous) et l'axe horizontal (opposition latérale et opposition frontale). Mais pour Hjelmslev, les deux axes de la représentation spatiale ne peuvent pas constituer la propriété pertinente de la troisième dimension dans la mesure où les différentes oppositions de la deuxième dimension (présupposées en vertu de (3)) ne se combinent pas librement avec les termes de l'opposition de verticalité ${ }^{11}$. Par contre, Hjelmslev observe que l'opposition de verticalité - opposition dessus / dessous - est toujours perçue de manière objective alors que l'opposition frontale devant-derrière fait intervenir la subjectivité, parce qu'elle tient compte de la place du locuteur dans l'évaluation du rapport entre la cible et le site ${ }^{12}$. Il y a donc prise en

10. $\mathrm{V}=$ reduplication de la voyelle précédente. Les autres suffixes de l'illatif sont : $-h V n$, -seen, -siin.

11. Selon Hjelmslev, un système casuel à trois dimensions qui épuiserait toutes les possibilités contiendrait 216 cas.

12. Hjelmslev donne en exemple les prépositions de lieu des langues germaniques qui présentent une opposition de cohérence - incohérence pour l'un des deux termes
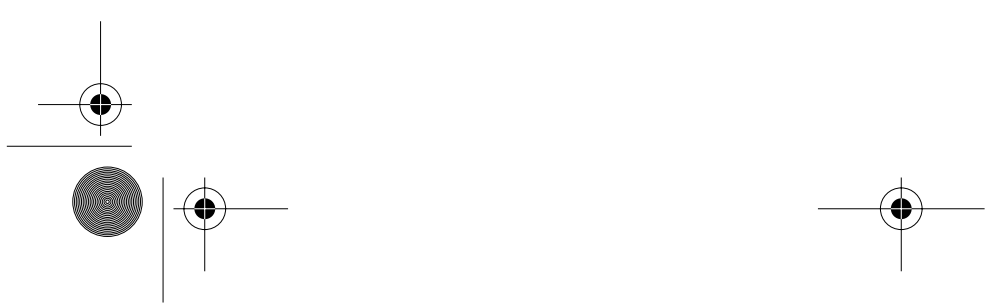
compte d'une propriété distinctive pour la troisième dimension qui relève d'une différence entre point de vue objectif et point de vue subjectif.

Les trois dimensions sont présentes en tabassaran, langue caucasique du Nord-Est. Le tableau (8) ne contient que les cas locatifs ( 40 cas). Le tabassaran compte au total 52 cas. La troisième dimension est ici représentée par la superposition de deux tableaux de cas locatifs à deux dimensions.

(8) Cas locatifs du tabassaran (trois dimensions) (voir tableau ci-contre)

La troisième dimension du tabassaran est justifiée par la présence d'une opposition entre un suffixe casuel qui exprime un rapport objectif (subessif) et un autre, un rapport subjectif (postessif). Pour être plus précis, l'emploi du subessif (9a) et du postessif en (9b) introduit trois informations: 1) la direction (pas de mouvement), 2) la nature du rapport entre la cible et le site (cohérence), et 3) l'orientation spatiale conçue objectivement (opposition dessus/dessous) ou subjectivement (opposition devant/derrière) :
(9) a.
calik $^{13}$
b. caliq
mur+SUBESSIF
mur+POSTESSIF
«sous le mur"
"derrière le mur»

Ces trois informations sont données en contexte dans l'exemple de Hjelmslev (10). Dans cette phrase, le verbe "vivre» est accompagné d'un complément locatif dont le suffixe casuel exprime 1) l'absence de mouvement, 2) une relation spatiale de cohérence (le rapport entre les poissons et leur milieu correspond plus précisément à ce que Hjelmslev désigne sous le terme d'inhérence ${ }^{14}$ ), et un rapport vertical dessus - dessous. Comme le montre la traduction de l'exemple, les deux prépositions indiquent une neutralisation en français d'une opposition pertinente en tabassaran. En français, on choisit l'une ou l'autre préposition selon la dimension retenue:

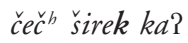

$$
\begin{aligned}
& \text { poissons eau+SUBESSIF vivent }
\end{aligned}
$$

«Les poissons vivent dans/sous l'eau »

Deux commentaires à propos de cette typologie : 1) Contrairement à ce que dit Hjelmslev de la relation entre la troisième dimension et les deux autres (la subjectivité-objectivité présuppose la cohérenceincohérence et la cohérence-incohérence présuppose la direction), il

de l'opposition de verticalité mais pas pour le second : en allemand, il y a deux prépositions pour "dessus " auf et über, et une pour "dessous » unter.

13. Mel'čuk 1994.

14. Dans la typologie de Hjelmslev, la cohérence se subdivise en inhérence et adhérence. Voir le schéma (17)
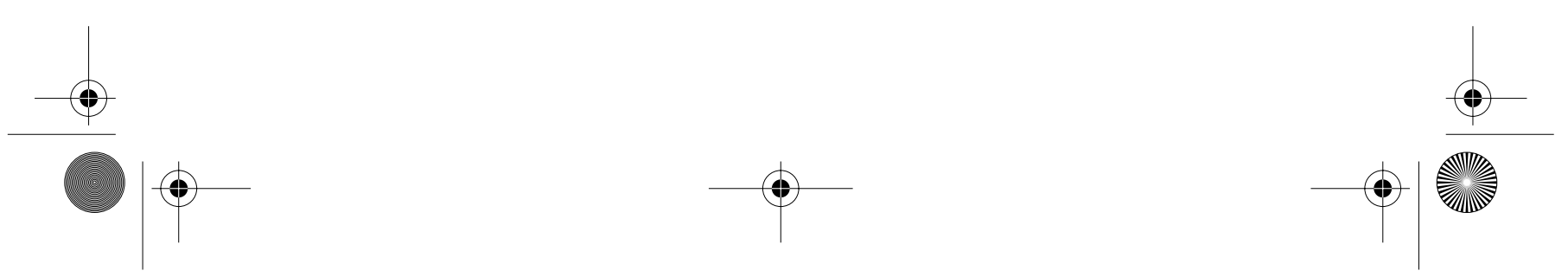


\begin{tabular}{|c|c|c|c|c|c|c|c|c|}
\hline & & & & & ence-in & hérenc & & \\
\hline & & $\begin{array}{r}\text { allati } \\
-\mathrm{hr}\end{array}$ & & & & & $\begin{array}{l}\text { ralatif } \\
\text { ilna }\end{array}$ & \\
\hline & & $\begin{array}{r}\text { adcomi } \\
-h\end{array}$ & & $\begin{array}{r}\text { instr } \\
\text { co }\end{array}$ & $\begin{array}{l}\text { nental- } \\
\text { tatif } \\
\text { ri }\end{array}$ & supra & $\begin{array}{l}\text { omitatif } \\
\text { ?ilri }\end{array}$ & $\begin{array}{r}\text { subcor } \\
-k\end{array}$ \\
\hline & . & $\begin{array}{r}\text { adver } \\
\text {-hir }\end{array}$ & & & $\begin{array}{l}\text { rsif II } \\
\text { adi }\end{array}$ & $\begin{array}{r}\text { supr } \\
-?\end{array}$ & $\begin{array}{l}\text { directif } \\
\text { lindi }\end{array}$ & $\begin{array}{r}\text { subv } \\
\text { kir }\end{array}$ \\
\hline & $!$ & $\begin{array}{r}\text { ablat } \\
\text {-ha }\end{array}$ & & & & & $\begin{array}{l}\text { latif } \\
\text { ilan }\end{array}$ & \\
\hline 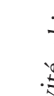 & & $\begin{array}{r}\text { adess } \\
-\mathrm{H}\end{array}$ & & & if II & & $\begin{array}{l}\text { eressif } \\
\text { ?il } \\
\end{array}$ & sub \\
\hline$\overline{\tilde{s}}$ & & & & hérence & ncohére & & & \\
\hline & & $\begin{array}{l}\text { llatif I } \\
-k^{\mathrm{h}} \mathrm{na}\end{array}$ & & $\begin{array}{l}\text { ifif I } \\
\text { ina }\end{array}$ & & & $\begin{array}{l}\text { postl } \\
-q^{h_{-}}\end{array}$ & \\
\hline & & $\begin{array}{l}\text { omitatif I } \\
-\mathrm{k}^{\mathrm{h}} \mathrm{ri}\end{array}$ & & $\begin{array}{l}\text { mitatif } \\
\text { iri }\end{array}$ & interc & $\begin{array}{l}\text { nitatif } \\
\text { i }\end{array}$ & $\begin{array}{r}\text { postcor } \\
\mathrm{q}^{\mathrm{h}}-\end{array}$ & $\begin{array}{l}\text { itatif } \\
\text { i }\end{array}$ \\
\hline . & $i^{\prime}$ & $\begin{array}{l}\text { liversif I } \\
\mathrm{k}^{\text {hindi }}\end{array}$ & & $\begin{array}{l}\text { ersif I } \\
\text { ndi }\end{array}$ & $\begin{array}{r}\text { inter } \\
-8\end{array}$ & $\begin{array}{l}\text { rectif } \\
\text { di }\end{array}$ & $\begin{array}{r}\text { postdi } \\
-\mathrm{q}^{\mathrm{h}-\mathrm{i}}\end{array}$ & $\begin{array}{l}\text { ectif } \\
\text { di }\end{array}$ \\
\hline & & $\begin{array}{l}\text { blatif I } \\
-k^{\text {han }}\end{array}$ & & tif I & ablatif- & $\begin{array}{l}\text { teressif } \\
n\end{array}$ & $\begin{array}{r}\text { ablatif-p } \\
-q^{\text {h- }}\end{array}$ & $\begin{array}{l}\text { stessif } \\
\text { in }\end{array}$ \\
\hline & . & $\begin{array}{l}\text { lessif I } \\
-\mathrm{k}^{\mathrm{h}}\end{array}$ & & $\begin{array}{l}\text { ssif I } \\
-\uparrow\end{array}$ & int & ssif & $\begin{array}{r}\text { poste } \\
-\mathrm{q}\end{array}$ & \\
\hline
\end{tabular}


semble bien que la subjectivité puisse être combinée directement à la direction. En yavapai - langue amérindienne d'Arizona - le locatif a deux formes ${ }^{15}$ selon la position du locuteur relativement au site et à la cible. Les deux phrases suivantes ont la même traduction mais la première phrase (avec locatif en $-k$ ) signifie que le locuteur se trouve en dehors de la maison, alors que la seconde phrase (avec locatif en $-m$ ) signifie qu'il est à l'intérieur de la maison:

(11) a. $\quad$ iñwāk vō̌ckiñ

1SG+maison+LOCATIF-1 sont sortis

«Ils sont sortis de ma maison» (locuteur hors de la maison)

b. $\quad$ กnwam vōčkiñ

1SG+maison+LOCATIF-2 sont sortis

«Ils sont sortis de ma maison» (locuteur dans la maison)

Pour finir, notons que les trois dimensions de la typologie de Hjelmslev se rapportent à trois modalités linguistiques: les oppositions de direction de la première dimension relèvent notamment des propriétés lexicales des items qui régissent les cas locatifs, les oppositions de la deuxième dimension (cohérence-incohérence) relèvent de la prédication syntaxique, et les oppositions de la troisième dimension (subjectivité-objectivité) relèvent quant à elles de l'énonciation. Dans ce qui suit, il ne sera question que des deux premières dimensions.

\section{Traits morphologiques des cas locatifs du finnois}

Une description du système des cas locatifs du finnois à partir de traits morphologiques permet de rendre compte des deux dimensions du système, des régularités lexicales et d'isoler dans les cas locatifs ce qui appartient à la prédication syntaxique. Dans cette approche en traits, chaque suffixe casuel est représenté par une matrice de traits non hiérarchisés : trois traits distinctifs et un trait commun qui oppose les cas locatifs à tous les autres cas. La liste des traits morphologiques est la suivante $\{ \pm$ LOCATIF, \pm LATIF, \pm ESSIF, \pm INTERNE $\}$. Le système d'oppositions est donné par le tableau (12) et chaque suffixe casuel est représenté par une matrice de traits en (13):

15. Cette langue n'a que deux cas locatifs (six cas en tout). La description de Mel'čuk 1994 s’appuie sur les données de Kendall 1975.
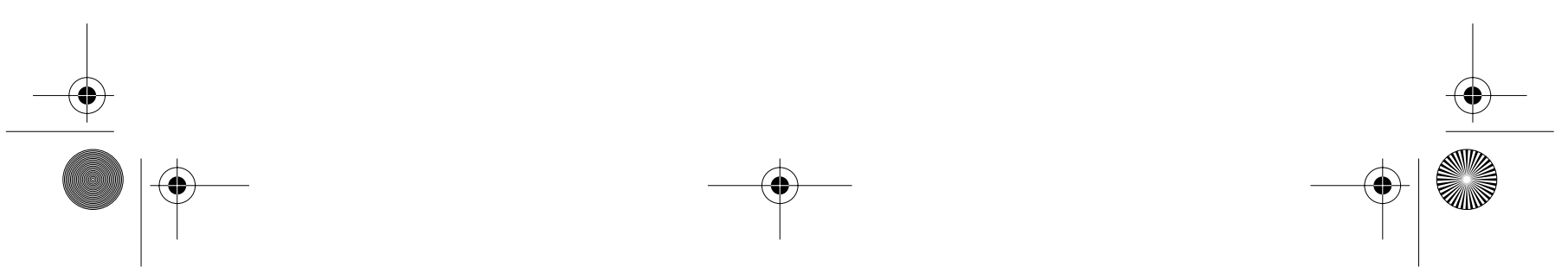


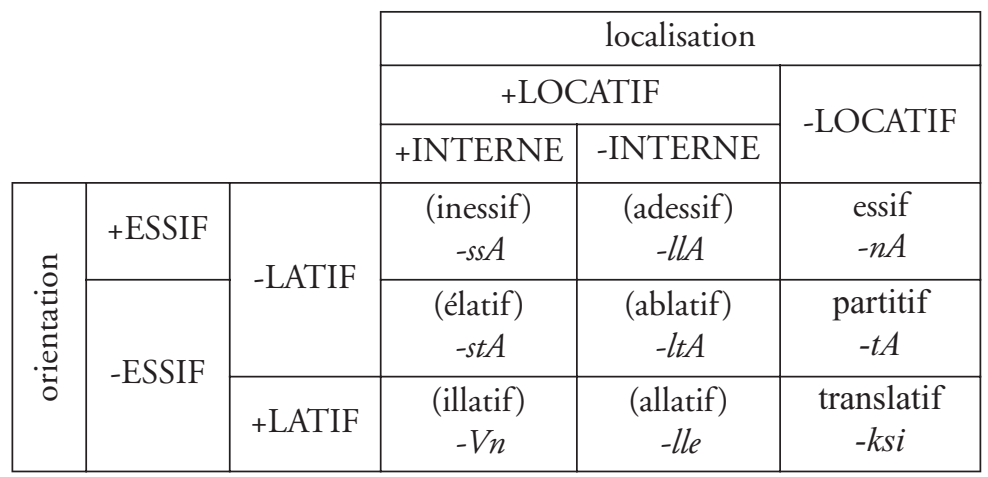

(12) Système des cas locatifs du finnois

$$
\begin{aligned}
& \text { inessif : }\left[\begin{array}{l}
+ \text { LOCATIF } \\
+ \text { INTERNE } \\
+ \text { ESSIF } \\
- \text { LATIF }
\end{array}\right] \\
& \text { adessif : }\left[\begin{array}{l}
+ \text { LOCATIF } \\
- \text { INTERNE } \\
+ \text { ESSIF } \\
- \text { LATIF }
\end{array}\right] \\
& \text { essif : }\left[\begin{array}{l}
- \\
\pm \\
\pm \\
+ \\
+ \text { INTSSIERNE } \\
- \text { LATIF }
\end{array}\right] \\
& \text { élatif: }\left[\begin{array}{l}
+ \text { LOCATIF } \\
+ \text { INTERNE } \\
- \text { ESSIF } \\
- \text { LATIF }
\end{array}\right] \\
& \text { ablatif : }\left[\begin{array}{l}
+ \text { LOCATIF } \\
- \text { INTERNE } \\
- \text { ESSIF } \\
- \text { LATIF }
\end{array}\right] \\
& \text { partitif : }\left[\begin{array}{l}
- \text { LOCATIF } \\
\pm \text { INTERNE } \\
- \text { ESSIF } \\
- \text { LATIF }
\end{array}\right] \\
& \text { illatif: }\left[\begin{array}{l}
+ \text { LOCATIF } \\
+ \text { INTERNE } \\
- \text { ESSIF } \\
+ \text { LATIF }
\end{array}\right] \\
& \text { allatif: }\left[\begin{array}{l}
+ \text { LOCATIF } \\
- \text { INTERNE } \\
- \text { ESSIF } \\
+ \text { LATIF }
\end{array}\right] \quad \text { translatif : }\left[\begin{array}{l}
- \text { LOCATIF } \\
\pm \text { INTERNE } \\
- \text { ESSIF } \\
+ \text { LATIF }
\end{array}\right]
\end{aligned}
$$

(13) Matrices de traits morphologiques des cas locatifs

Dans le tableau (12), Les termes "orientation» et «localisation» remplacent respectivement les termes «direction» et "cohérence-incohérence " de la typologie de Hjelmslev. D'une part, "orientation " est préférable à "direction », car ce premier terme, contrairement au second, n'est pas lié au mouvement ${ }^{16}$. D'autre part, «localisation» est un terme plus général pour désigner la seconde dimension des systèmes casuels, car il ne comporte aucune interprétation, aucune analyse particulière, quant à l'ontologie de la relation entre la cible et le site. Les définitions suivantes sont empruntés à Mel'čuk ${ }^{17}$. La définition de l'orientation

16. Je considère ici qu'en l'absence de mouvement, la cible et le site sont des objets orientés du fait de la relation asymétrique entre les deux objets (le site est une source ou une destination possible pour un mouvement de la cible).

17. Mel'čuk 1994. 
est légèrement modifiée (texte entre crochets) afin d'y inclure les cas statiques.

(14) Orientation ${ }^{18}$ : catégorie dont les éléments spécifient la direction (ou l'absence de direction) du déroulement d'un événement par rapport à l'objet en question.

Localisation : catégorie dont les éléments spécifient la position spatiale d'un objet ou d'un fait quelconque par rapport à l'objet ou (moins souvent) au fait en question en termes de configuration géométrique.

Le trait [+LOCATIF] permet d'isoler les cas locatifs parmi l'ensemble des cas. On remarquera que le tableau (12) intègre trois cas non locatifs - l'essif, le translatif et le partitif - qui ont une valeur locative uniquement dans des formes figées adverbiales et postpositionnelles. Il s'agit notamment de l'adverbe ulko- "dehors" et de la postposition luo- «chez». Lorsque ces mots sont à l'essif, au partitif ou au translatif, ils participent à l'orientation dans les mêmes conditions que les autres cas locatifs mais sans référence à la localisation. Quelques exemples :

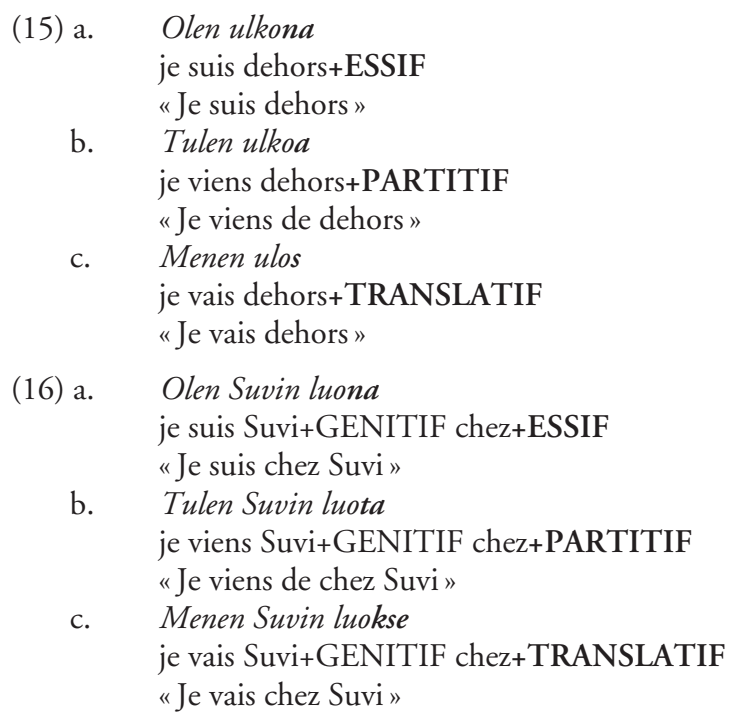

Le trait $[ \pm$ INTERNE] s'applique à la seconde dimension (la localisation); le trait [+INTERNE] renvoie à la cohérence et le trait [INTERNE] à l'incohérence de la typologie de Hjelmslev. La cohérence recouvre l'inhérence et l'adhérence. Hjelmslev propose les schémas suivants pour expliciter les différents rapports spatiaux correspondant à la

18. L'orientation dont il est question ici est l'orientation II de Mel'čuk 1994, l'orientation I étant définie par référence au locuteur.
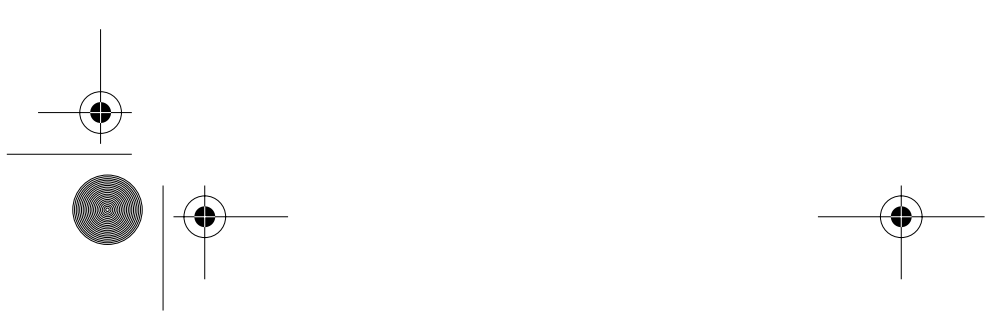
localisation. Ces schémas sont légèrement modifiés de façon à y inclure les termes de cible et de site :

(17) Opposition entre cohérence et incohérence, d'après Hjelmslev ${ }^{19}$

\begin{tabular}{|c|c|}
\hline $\begin{array}{c}\text { cohérence } \\
\text { (cas internes) }\end{array}$ & $\begin{array}{c}\text { incohérence } \\
\text { (cas internes) }\end{array}$ \\
\hline site & cible
\end{tabular}

Inhérence et adhérence représentent les termes marqués de l'opposition de seconde dimension. Dans un rapport d'inhérence, la cible est incluse dans le site et dans un rapport d'adhérence, la cible est dans une relation de contact qui exclut la relation porteur / porté ${ }^{20}$. L'adhérence ne recouvre donc pas les emplois des cas internes pour un objet posé sur une surface horizontale ${ }^{21}$. Les exemples (18a-c) avec l'inessif (absence de mouvement) illustrent respectivement l'inhérence (18a) et l'adhérence (18b-c) :

(18) a.

$$
\begin{aligned}
& \text { Suvi on saunassa } \\
& \text { Suvi est sauna+INESSIF } \\
& \text { "Suvi est dans le sauna» }
\end{aligned}
$$
b. Lamppu on katossa lampe est plafond+INESSIF
"La lampe est au plafond"

(inhérence)

(adhérence)

19. Hjelmslev 1935.

20. Vandeloise 1986.

21. Dans les cas mixtes - verticalité et faible adhérence - les deux séries casuelles sont possibles. Exemples (Ikola 1983) : kirjoitetaan tauluun (illatif) «on écrit sur le tableau » et kirjoitetaan taululle (allatif) «on écrit au tableau».
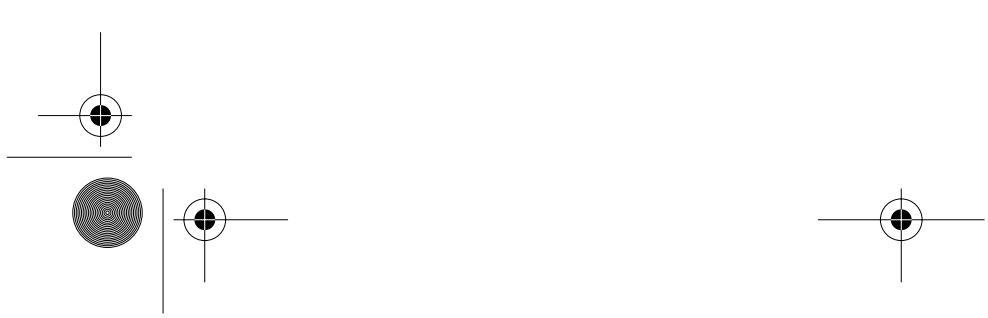


\section{c. Huivi on kaulassa (adhérence) \\ foulard est cou+INESSIF \\ «Le foulard est autour du cou»}

Les emplois des cas externes ${ }^{22}$ les plus significatifs sont ceux où le site est un espace ouvert (19a), un lieu perçu comme repérage et non comme un espace ou un contour - sa fonction l'emporte sur ses propriétés géométriques - (19b), et une surface de contact horizontal (19c):

$\begin{array}{rlr}\text { (19) a. } & \begin{array}{l}\text { Suvi on kadulla } \\ \text { Suvi est rue+ADESSIF } \\ \text { "Suvi est dans la rue» }\end{array} & \text { (incohérence) } \\ \text { b. } & \text { Suvi on asemalla (incohérence) } & \\ & \text { Suvi est gare+ADESSIF } \\ & \text { "Suvi est à la gare» } & \\ \text { c. } & \text { Kirja on pöydällä } & \\ & \text { livre est table+ADESSIF } & \\ & \text { "Le livre est sur la table» } & \text { (incohérence) }\end{array}$

Les deux autres traits $([ \pm \mathrm{LATIF}]$ et $[ \pm \mathrm{ESSIF}])$ décrivent l'orientation. Le trait $[ \pm \mathrm{ESSIF}]$ oppose le mouvement et l'absence de mouvement. Le trait $[ \pm \mathrm{LATIF}]$ permet de distinguer la direction de manière asymétrique: rapprochement ([+LATIF]) vs non rapprochement ([-LATIF]). Le non rapprochement inclut l'absence de mouvement et l'éloignement. Le trait [-LATIF] regroupe des cas pour lesquels il n'y a pas d'unité sémantique et qui se définissent par conséquent de manière négative. Le trait [-LATIF] a cependant une justification sur le plan de l'expression et du contenu. La forme des cas locatifs [-LATIF] s'explique par la diachronie $:^{24}$ les quatre cas locatifs correspondant à ce trait (inessif, élatif, adessif et ablatif) sont réguliers dans leur forme et peuvent être analysés comme des cas composés ${ }^{25}$ c'est à dire comme des suffixes casuels décomposables en deux parties: le premier segment est un suffixe

22. Le contraste suivant emprunté de nouveau à Ikola 1983 fournit un exemple simple d'opposition minimale entre adhérence (cas interne) et incohérence (cas externe) : (i) ilmoitus naulataan oveen (illatif) "on a cloué l'annonce sur la porte», (ii) ilmoitus naulataan ovelle (allatif) "On a cloué l'annonce à la porte». En (ii) l'emploi du cas externe allatif signifie simplement que l'annonce est à l'extérieur de la maison (le nom porte est alors interprété comme ce qui délimite l'intérieur de l'extérieur); l'annonce peut très bien être apposée à côté de la porte.

23. Voir la note 5 pour l'interprétation de cette phrase.

24. Hakulinen 1968.

25. Mel'čuk 1994.

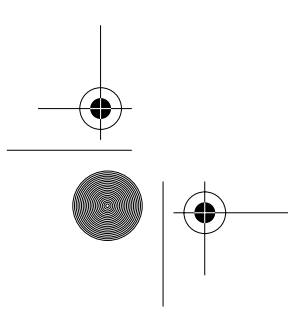


de localisation $\left({ }^{*}-s\right.$ pour le trait [+INTERNE] et ${ }^{*}-1$ pour le trait [INTERNE]) tandis que le second segment est le suffixe d'essif $(-n A)$ ou de partitif $(-t A) .{ }^{26}$ Cette analyse en termes de cas composés ne s'applique pas aux deux suffixes [+LATIF] (illatif et allatif). Le regroupement des quatre cas [-LATIF] se justifie également au niveau conceptuel; l'unique différence entre l'inessif et l'élatif (de même entre l'adessif et l'allatif) tient à l'opposition entre mouvement et absence de mouvement. Si l'on privilégie le moment initial du déplacement exprimé par l'élatif et l'ablatif, le rapport spatial entre la cible et le site des cas [-LATIF] est alors le même: il s'agit d'un rapport qui s'interprète dans le cadre de la localisation (opposition cohérence/incohérence). Les deux cas opposés, illatif et allatif représentent quant à eux un rapport spatial initial distant. Ces deux propriétés de l'opposition [ $[ \pm$ LATIF] sont résumées par les schémas suivants:

(20) Propriété commune des cas [-LATIF] (cas internes)

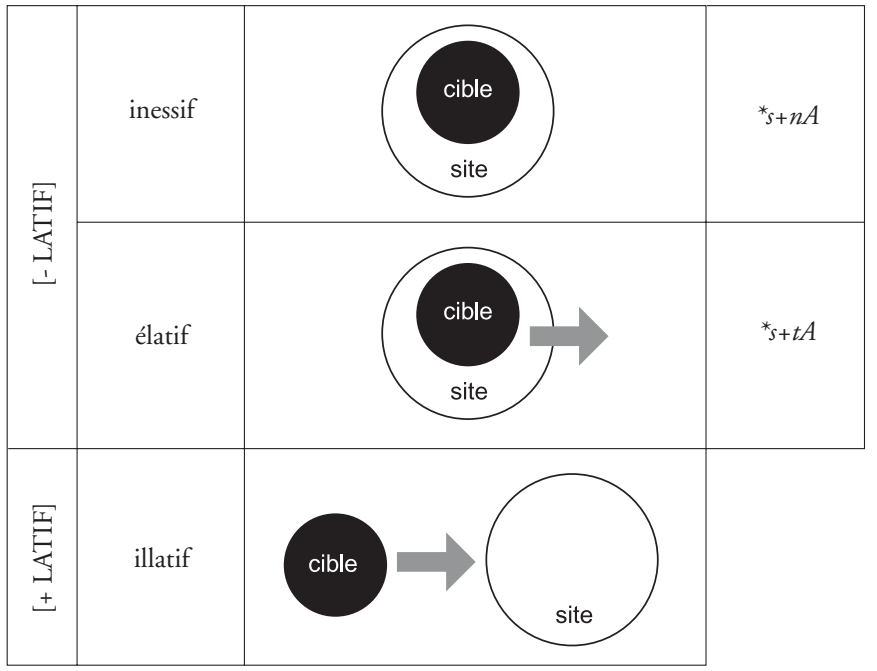

26. $-s s A<--^{*} s+n A,-s t A<--^{*} s+t A,-l l A<--^{*} l+n A, l t A<-{ }^{*} l+t A$.
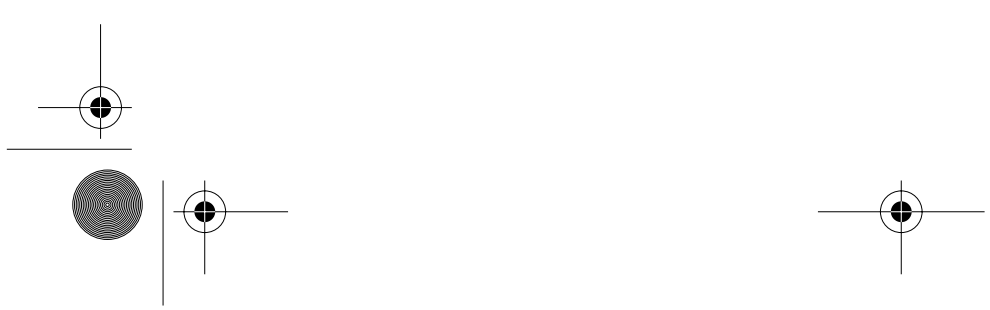
(21) Propriété commune des cas [-LATIF] (cas externes)

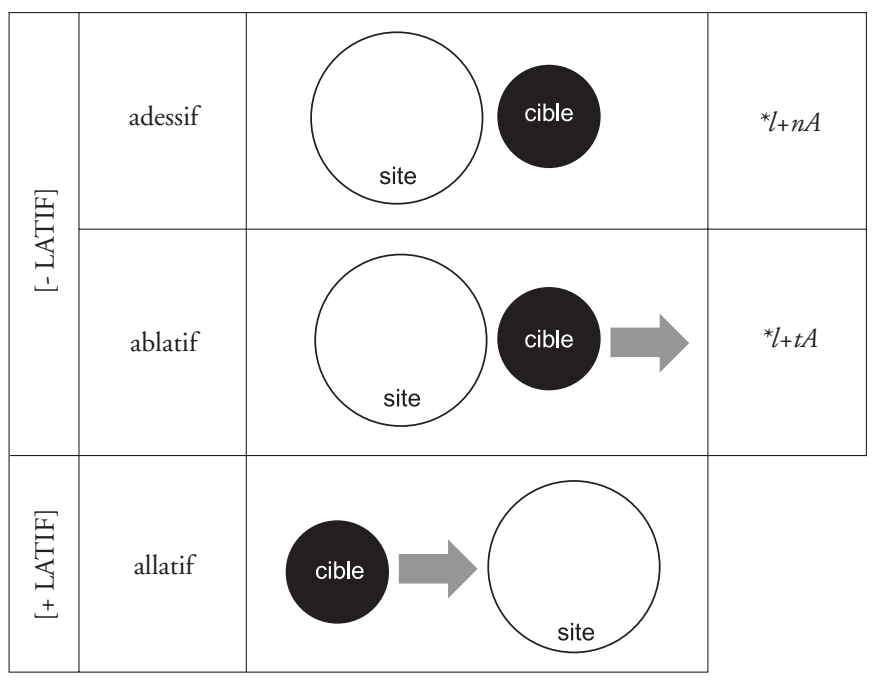

En résumé, les oppositions conceptuelles pertinentes pour le système des cas locatifs sont les suivantes:

(22) - mouvement $v$ s absence de mouvement $([ \pm \mathrm{ESSSIF}])$

- proximité vs distalité ([ $[\mathrm{LATIF}])$

- cohérence $v s$ incohérence $([ \pm I N T E R N E])$

Les traits morphologiques ne présentent pas seulement un intérêt sur le plan descriptif mais également sur le plan explicatif dans la mesure où ils permettent de rendre compte de régularités sémantiques dans le lexique. Seuls ou combinés, ils participent à la sous catégorisation. Pour illustrer ce point, considérons les exemples (23a-b). Le verbe mennä «aller» a un complément locatif qui peut être soit à l'illatif soit à l'allatif:

(23) a. Menen pankkiin

je vais banque+ILLATIF

Je vais à la banque »

b. Menen kadulle

je vais rue+ALLATIF

Je vais dans la rue»

Il est clair que si la sous-catégorisation du verbe fait explicitement référence à ces deux cas comme l'indique la représentation lexicale simplifiée (24a), la description n'est pas minimale puisqu'il y a conjointement une propriété redondante et une propriété non pertinente. Dans les exemples (23a-b), la redondance concerne l'illatif et l'allatif: tous les deux font référence à un mouvement de rapprochement. Par ailleurs,
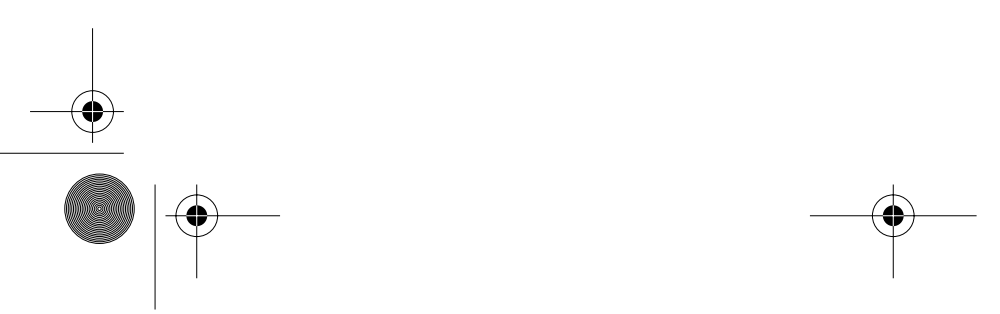
les deux suffixes casuels font référence à la localisation, alors même que cette propriété ne relève pas de la sous- catégorisation du verbe, car aucune propriété sémantique du verbe ne permet de départager l'illatif de l'allatif dans le lexique. En recourant au trait [+LATIF], on évite la redondance et on élimine du lexique l'opposition entre cas interne (illatif) et cas externe (allatif), qui s'avère inutile à la caractérisation du verbe. La représentation (24b) est donc plus concise et plus fidèle à une conception de l'information lexicale réduite aux seules propriétés inhérentes aux têtes lexicales :

(24) a. mennä: __ ILLATIF/ALLATIF ${ }^{27}$
"aller»
b. mennä: _
"aller» ${ }^{\text {LLATIF }]}$

Un autre exemple de représentation lexicale minimale au moyen des traits morphologiques est proposé en (25): Le verbe lainata, qui signifie aussi bien " emprunter " que "prêter ", admet quatre cas différents. Sa sous-catégorisation, reformulée avec les traits casuels, contient donc uniquement le trait [-ESSIF] :

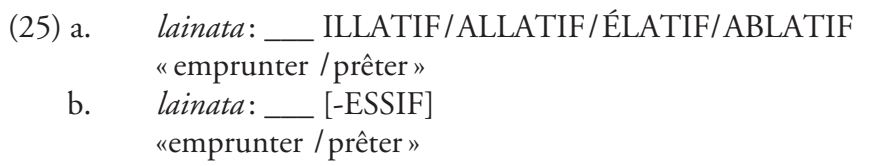

Une recherche effectuée sur une base de données d'environ 500 verbes $^{28}$ a permis de dégager un échantillon significatif de classes verbales. Les classes suivantes correspondent aux verbes dont la souscatégorisation locative peut être exprimée au moyen d'un seul trait:

(26) a. [+LATIF] : \{antad "donner", istuutua "s'asseoir", kutsua « inviter», soittaa « téléphoner», sopia « convenir »...

b. [-LATIF] : $\{$ poiketa " dévier "\}

c. [+ESSIF] : \{asua "résider", käydä «se promener", odottaa « attendre ", pysyä « rester ", tavata « rencontrer »... $\}$

d. [-ESSIF] : \{astua « monter", jatkua « continuer», kuulua " appartenir », pudota " tomber », vied $\ddot{a}$ «apporter /emporter »...\}

e. $\quad[+\mathrm{LOCATIF]}:\{j a ̈ a ̈ d \ddot{a}$ " rester », näky $\ddot{a}$ « être visible»... $\}$

La base de données ne contient qu'un seul verbe sous-catégorisé pour le trait [-LATIF]. Il n'y a là rien de surprenant étant donné que

27. Cette notation indique que le verbe mennä "aller» prend un complément locatif à l'illatif ou à l'allatif.

28. Cette base de données a été élaborée à partir des informations contenues dans l'ouvrage sur la rection des mots finnois de Jönsson-Korhola et White 1997. 
la classe définie par ce trait apparaît comme la moins naturelle dans le système d'oppositions retenu pour la description des cas locatifs.

La sous-catégorisation d'un verbe peut contenir une matrice casuelle complète, comme dans l'exemple (27). Le verbe iloita «se réjouir " n'accepte qu'un complément à l'élatif. Mais cette observation ne remet pas en cause les généralisations précédentes car il s'agit d'un complément de cause et non d'un complément locatif ${ }^{29}$ :

(27) a.

$$
\text { iloita :__ ÉLATIF (cause) }
$$

«se réjouir»

b.

$$
\text { iloita }:-\left[\begin{array}{l}
+ \text { LOCATIF } \\
+ \text { INTERNE } \\
- \text { ESSIF } \\
- \text { LATIF }
\end{array}\right] \text { (cause) }
$$

Cette recherche sur un échantillon d'environ 500 verbes ayant un complément locatif permet de dégager les observations suivantes ${ }^{30}$ :

(28) a. Aucun verbe n'est sous-catégorisé exclusivement pour une valeur de localisation. Autrement dit, aucun verbe n'a pour unique trait de souscatégorisation le trait [+INTERNE] ou [-INTERNE].

b. Le trait de localisation $[ \pm I N T E R N E]$ n'entre pas dans la sous-catégorisation des verbes avec complément(s) locatif(s).

Ces observations excluent par conséquent d'avoir des représentations lexicales telles que (29a-b) :

(29) a. *V: $+[+\mathrm{INTERNE}]$

b. $\quad{ }^{*} \mathrm{~V}$ : $+[$-INTERNE]

29. La cause est toujours exprimée par l'élatif. L'opposition entre cas interne et cas externe est donc impossible. Seule une interprétation localiste forte serait à même de régulariser l'ensemble des valeurs sémantiques des cas locatifs. La systématique proposée ici ne concerne que les emplois spatiaux, voire temporels (mise à part quelques oppositions du type päivässä "en un jour» (durée) päivälllä «le jour», l'opposition entre cas internes et cas externes des compléments de lieu n'est pas aussi systématique pour les compléments de temps).

30. Ces observations découlent des résultats suivants: a) aucun verbe ne sélectionne les trois cas de l'une des deux séries (cas internes et cas externes) à l'exclusion des trois autres, b) si un verbe sélectionne un cas de l'une des deux séries, alors il sélectionne le cas correspondant de l'autre série.
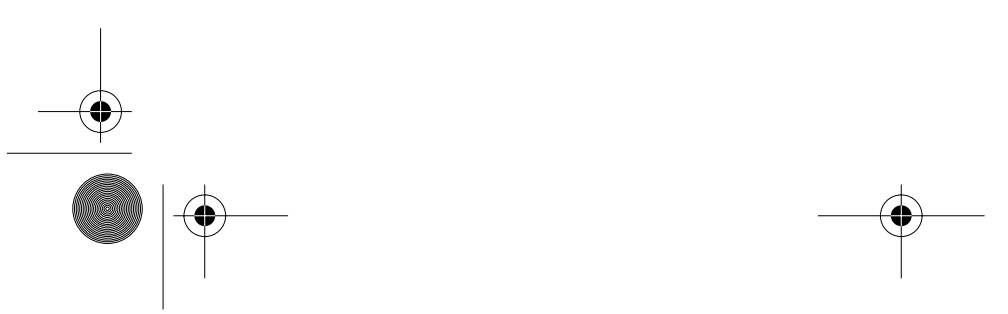
Ce résultat est à mettre en rapport avec cette autre donnée : il n’y a pas en finnois deux verbes qui s'opposent par la localisation. À titre d'exemple, la différence entre les verbes français mettre et poser n'est pas en finnois d'ordre lexical mais syntaxique; le verbe panna «mettre / poser" a pour seul trait de sous-catégorisation le trait d'orientation [+LATIF] (30a). Le choix entre cas interne (30b) et cas externe (30c) est fixé en syntaxe par la nature du rapport spatial entre la cible et le site. Les exemples (30d-e) illustrent les deux schémas syntaxiques (30b-c) :

(30) a.

panna: [+LATIF]

«mettre/poser»

b. panna $+[\mathrm{SN} \cdots+$ ALLATIF $]=$ «mettre sur/poser »

c. panna $+[\mathrm{SN} \cdots+$ ILLATIF $]=$ «mettre dans »

d. Suvi a pani kirjat pöydälle

Suvi a mis les livres table+ALLATIF

"Suvi a mis/posé les livres sur la table»

e. Suvi pani kirjat laatikkoon

Suvi a mis les livres tiroir+ILLATIF

"Suvi a mis les livres dans le tiroir"

Dans le même ordre d'idée, le verbe français «entrer» n'a pas d'équivalent lexical en finnois. Son équivalent syntaxique est un verbe de mou-

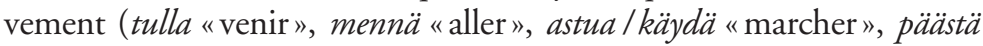
"pouvoir entrer »...) suivi de la postposition locative sisä- «à l'intérieur de" à un cas locatif de rapprochement (illatif ou allatif). Exemples: tulla sisä̈̈n, tulla sisälle « entrer ".

La sous-catégorisation n'étant pas limitée aux verbes, il est possible d'étendre les observations (28) à d'autres catégories. Je propose par conséquent l'hypothèse suivante :

(31) La sous-catégorisation d'un complément locatif ne contient aucune référence à la localisation mais uniquement des traits d'orientation ([ \pm ESSIF $]$, $[ \pm$ LATIF] $)$.

Cette version étendue de (28) permet de rendre compte notamment $\mathrm{du}$ jeu entre cas internes et cas externes des deux postpositions qui prennent un complément locatif (lieu et temps) asti et saakka «depuis/à partir de / jusqu'à »"

(32) a. pohjoisnavalta asti «à partir du pôle nord» (ablatif)

b. pohjoisnavalle asti « jusqu'au pôle nord" (allatif)

c. Amerikasta asti "à partir de l'Amérique" (élatif)

d. Amerikkaan asti «jusqu'en Amérique» (illatif)

31. Ces postpositions étant également non spécifiées pour la direction, leur trait de sous-catégorisation est [-ESSIF]. 
e. 1970-luvulta asti « depuis les années 70 » (ablatif)

f. 1970-luvulle asti « jusqu'aux années 70 » (allatif)

g. aamusta asti « depuis le matin» (élatif)

h. aamuun asti " jusqu' au matin» (illatif)

(33) a. rannikolta saakka « à partir de la côte (ablatif)

b. rannikolle saakka "jusqu'à la côte" (allatif)

c. Suomesta saakka "à partir de la Finlande» (élatif)

d. Suomeen saakka "jusqu'en Finlande» (illatif)

e. 1970-luvun loppupuoleltasaakka "depuis la fin des années 70 " (ablatif)

f.1970-luvun loppupuolellesaakka "jusqu'à la fin des années 70 " (allatif)

g. vuodesta 1990 saakka "depuis 1990 » (élatif)

h. vuoteen 1990 saakka «jusqu'en 1990 » (illatif)

Un autre argument en faveur du caractère non régi de la localisation est fourni par le locatif des noms de lieux (villes, pays, régions naturelles...). Ces derniers sont soit à un cas interne (le plus souvent) soit à un cas externe, et cette répartition est en partie arbitraire ${ }^{32}$. Le noms des villes suivantes se décline avec les cas externes \{Tampere, Imatra, Rauma, Loimaa...\}, la même chose pour les noms de pays ou de régions naturelles suivants \{Pohjanmaa (Ostrobothnie), Venäjä (Russie), Balkan (Balkans), Krim (Crimée)...\}.

\section{Localisation et prédication syntaxique}

Résumons les différents points développés jusqu'ici: les cas locatifs du finnois sont un système à deux dimensions (orientation et localisation). L'orientation est à mettre en rapport avec les propriétés de sous-catégorisation des items lexicaux alors que la localisation concerne la nature de la relation spatiale entre la cible et le site. L'analyse des cas locatifs sous la forme d'une matrice de traits morphologiques permet de rendre compte de ce système à deux dimensions en associant chacune d'elles à un ou plusieurs traits : l'orientation est représentée par la combinaison des traits $\left[{ }_{ \pm} \mathrm{ESSIF}\right]$ et $\left[{ }_{ \pm} \mathrm{LATIF}\right]$, tandis que la localisation est représentée par le trait $[ \pm$ INTERNE]. Les traits d'orientation concourent à la caractérisation des items lexicaux sous-catégorisés pour un complément locatif en ne donnant comme information syntaxique que le

32. Pour les noms des îles, c'est plutôt l'inverse; bien que les îles se distinguent des autres lieux géographiques par leur délimitation naturelle, les cas externes sont plus fréquents. On observe plusieurs cas de figures : 1) les noms d'îles au pluriel (Antillit, Baleaarit, Filippiinit, Malediivit, Seychellit...), ainsi que les noms des îles formés sur le mot maa "terre" (Ahvenanmaa, Hiidenmaa, Saarenmaa...) ont un cas externe, 2) d'autres (Kreeta, Kuuba, Ruissalo, Sardinia, Sisilia,...) prennent un cas interne, 3) et le reste (Ceylon, Jaava, Korsica, Kypros, Madagaskar, Madeira, Malta...) admet les deux, avec cependant une nette préférence pour les cas externes.
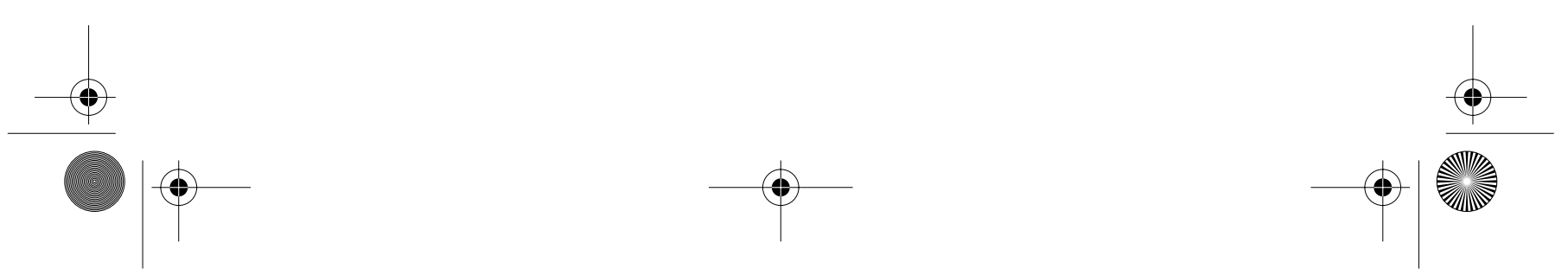
strict nécessaire. Par ailleurs, ce sont les traits d'orientation inclus dans la sous-catégorisation - et non les suffixes de cas - qui doivent être associés aux entités et primitives de la sémantique de Jackendoff ${ }^{33}$.

Dans le cadre du modèle de Jackendoff, la représentation sémantique des phrases avec complément locatif est obtenue en associant les traits d'orientation et de localisation à des expressions complexes constituées des entités [CHEMIN _- ] et [LIEU_- et des fonctions primitives $\{\mathrm{IN}, \mathrm{ON}, \mathrm{FAST}(\mathrm{EN}), \mathrm{NEAR}\}$. Les expressions référentielles (cible et site, notamment) sont quant à elles associées à l'entité [CHOSE _ ] . Les correspondances entre traits de cas locatifs et entités / fonctions sont les suivantes (les notions sémantiques utilisées précédemment sont mentionnées entre parenthèses $)^{34}$ :

(34) a. traits d'orientation

$[+$ ESSIF] $\rightarrow$ [LIEU IN/ON/FAST/NEAR_] (absence de mouvement)

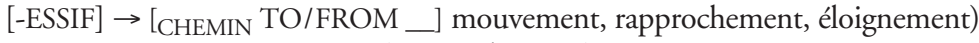

[+LATIF] $\rightarrow$ [CHEMIN TO _ $]$ (rapprochement)

[-ESSIF, -LATIF] $\rightarrow$ [CHEMIN FROM__] (éloignement)

b. traits de localisation

[+INTERNE] $\rightarrow$ [LIEU IN/FAST__] (cohérence, inhérence, adhérence, contact) [-INTERNE] $\rightarrow$ [LIEU ON/NEAR__] (incohérence, proximité)

Revenons à la sous-catégorisation. Conformément aux observations (28) et (31), les verbes et autres catégories qui prennent un complément locatif n'ont pour seule information que les traits d'orientation pour caractériser le ou les complément(s) locatif(s). Dans les représentations lexicales, les traits locatifs de sous-catégorisation sont associés - coïndicés - avec les entités / fonctions de la structure conceptuelle selon les correspondances données en (34a). À titre d'illustration, je donne en (35) les représentations lexicales des trois verbes présentés dans la précédente partie (mennä "aller», lainata «emprunter / prêter» et panna "mettre / poser»). Dans ces trois entrées lexicales, le trait d'orientation est coïndicé avec l'entité [CHEMIN _ $]^{35}$.

33. On trouvera dans Nikanne 1986 et Leino et al. 1990, une description des cas locatifs (sans décomposition en traits) dans le cadre de la sémantique de Jackendoff (1983, 1990). Dans François 1996, 2002, le système des cas locatifs du finnois est mis en parallèle avec ceux du hongrois et de l'estonien.

34. La correspondance entre fonctions primitives et traits de cas n'est pas biunivoque : un trait de cas peut avoir deux fonctions différentes et une fonction particulière peut correspondre à une combinaison de deux traits.

35. 1 = entrée lexicale (forme phonologique, thème, classe verbale...), 2 = catégorie, 3 = sous-catégorisation, $4=$ structure conceptuelle (représentation sémantique).
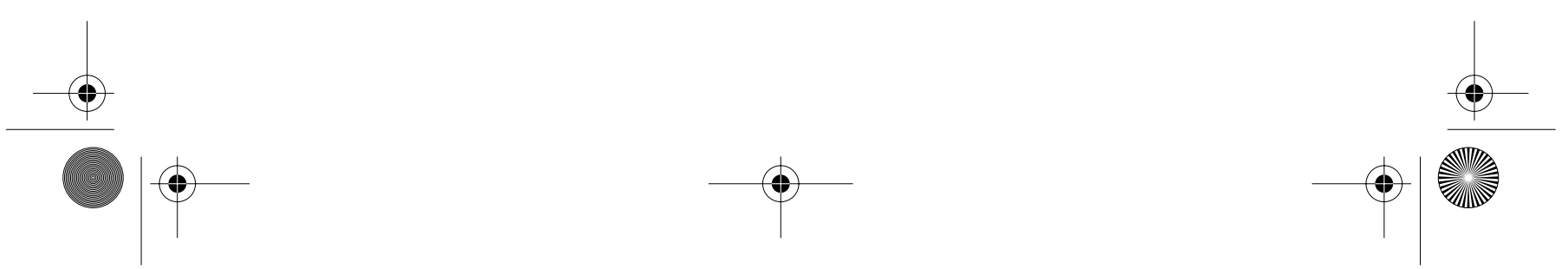
a.

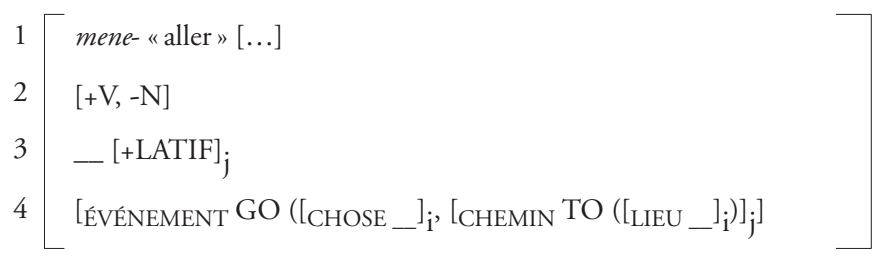

b.

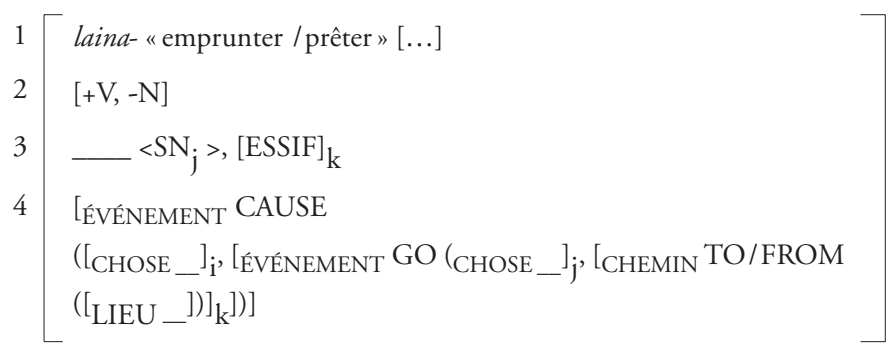

c.

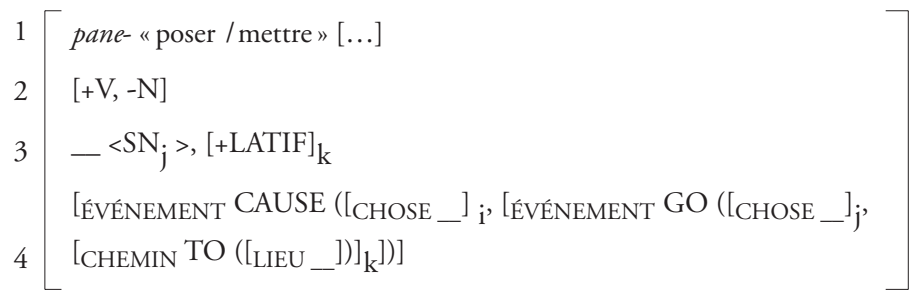

Bien que morphèmes liés, les suffixes de cas locatifs ont également une représentation lexicale complète. Deux exemples (allatif et illatif):

(36)

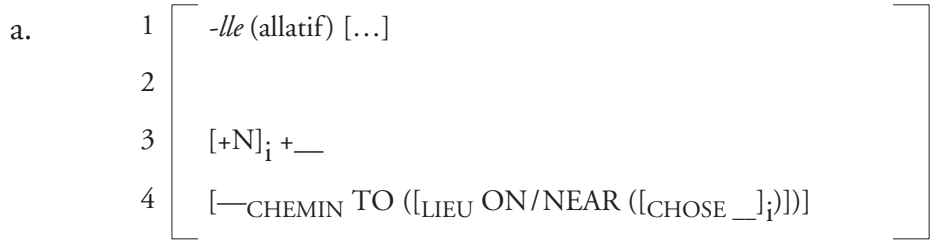
b. $\quad 1[-V n,-h V n,-$ seen (illatif) $[\ldots]$
$3 \quad[+\mathrm{N}]_{\mathrm{i}}+$
4 [CHEMIN TO $\left(\left[\right.\right.$ LIEU IN/FAST $\left.\left.\left.\left(\left[\text { CHOSE }_{\text {C }}\right]_{\mathrm{i}}\right)\right]\right)\right]$

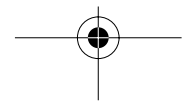


Considérons maintenant le contraste entre les phrases (30d) et (30e), reprisent ici :

(37) a.

Suvi pani kirjat pöydälle

Suvi a mis les livres table+ALLATIF

"Suvi a mis/posé les livres sur la table»

b. Suvi pani kirjat laatikkoon

Suvi a mis les livres tiroir+ILLATIF

«Suvi a mis les livres dans le tiroir»

Ces deux phrases qui s'opposent par le locatif (choix lexical et cas locatif) ont respectivement pour structure conceptuelle (38a) et (38b) :

(38) a.

[ÉvÉNEMENT CAUSE ([CHOSE Suv] ],

[ÉVÉNEMENT GO ([CHOSE kirjat], [CHeMIN TO ([LIEU ON ([CHOSE pöytä])]]])])]

b.

[ÉVÉNEMENT CAUSE ([CHOSE Suvi],

[ÉVÉNEMENT GO ([CHOSE kirjat], [CHEMIN TO ([LIEUIN ([CHOSElaatikko]])]]])]

Ces représentations sémantiques partielles, qui n'explicitent que les propriétés lexicales du prédicat verbal, sont les résultantes de la structure conceptuelle du verbe panna «mettre / poser » $(35 \mathrm{c})^{36}$ et des structures conceptuelles des suffixes locatifs (36a) et (36b). De par sa sous-catégorisation, l'insertion lexicale du verbe est à même d'expliquer l'occurrence de l'entité complexe [CHemin TO ([LIeU_ $]$ )] en (38). Par contre, l'insertion d'un suffixe casuel satisfaisant la sous-catégorisation verbale ([+LATIF]) ne permet pas d'expliquer la distribution du trait $[ \pm \text { INTERNE }]^{37}$. J'admettrai ici qu'un suffixe de cas locatif est soumis au principe suivant :

36. La représentation lexicale du verbe panna "mettre / poser" est incomplète en (35c) car elle n'intègre pas la possibilité pour ce verbe d'avoir un second complément locatif facultatif exprimant l'origine du déplacement. Exemple :

Suvi pani kirjat kassista pöydälle

Suvi a mis les livres sac+ÉLATIF table+ALLATIF

"Suvi a pris les livres dans le sac pour les mettre sur la table»

La sous-catégorisation complète de ce verbe est par conséquent : _ $<\mathrm{SN}>$, ([-ESSIF, -LATIF]), [+LATIF]. La structure conceptuelle doit être également enrichie afin d'y inclure l'entité / fonction de ce complément facultatif.

37. Pour les adverbes de lieu introduits en (15), la sous-catégorisation verbale suffit puisque ces adverbes ont un cas (essif, partitif ou translatif) dont la matrice de traits ne contient pas de trait [ \pm INTERNE]. Contrairement aux locatifs ordinaires, la nature de la relation spatiale entre la cible et le site est déterminée par les propriétés inhérentes de la tête lexicale de l'adverbe (ulko- «extérieur» dans les exemples 15a-c). 
(39) a. Les traits locatifs d'orientation et de localisation doivent être interprétés.

b. Les traits locatifs d'orientation et de localisation sont associés à des entités / fonctions de la structure conceptuelle.

Pour rendre compte de l'opposition entre (37a) et (37b), il convient de faire appel à la prédication syntaxique. À cet effet $\mathrm{j}$ ’introduirai en complément de l'hypothèse (31), cette seconde hypothèse majeure:

(40) La seconde dimension des cas locatifs (la localisation) relève de la prédication syntaxique.

Par prédication syntaxique ${ }^{38}$, il faut entendre la mise en relation de deux constituants syntaxiques autonomes (projections maximales) dont l'un est prédicat et l'autre son sujet de prédication. La relation prédicatsujet de prédication est de nature aristotélicienne; elle repose sur un jugement (dire quelque chose à propos de quelque chose) et n'admet d'un seul argument (le sujet de prédication) ${ }^{39}$, de plus, elle met en rapport des expressions référentielles (cible et site, pour ce qui nous intéresse ici) qui ne sont liées par aucune relation de dépendance lexicale ${ }^{40}$. De même que la relation entre un prédicat verbal et son sujet est sensible au contenu lexical des positions argumentales (le rôle thématique du sujet n'est pas le même dans Antoine a pris son parapluie (agent) et dans Antoine a pris une raclée (patient)), la relation de prédication entre la cible et le site des phrases avec complément locatif est sensible au choix lexical des termes engagés dans la relation. C'est précisément la nature du rapport spatial entre le prédicat locatif (le site) et son sujet de prédication (la cible) qui détermine la valeur du trait $[ \pm$ INTERNE] selon les propriétés ontologiques du site et de la cible rapportées aux modalités particulières du système des cas locatifs de la langue.

Les deux relations pertinentes pour expliquer la distribution des cas locatifs dans les deux exemples de référence (37a-b), sont isolées dans les schémas (41) et (42). La sous-catégorisation est mise en évidence en (41a-b) par le complexe verbal formé du verbe et du complément locatif, tandis que la prédication syntaxique est représentée en (41a-b) par la mise en relation de l'objet (sujet de prédication) et du prédicat

38. Rothstein 1985.

39. En cela, elle se distingue de la prédication frégéenne qui admet plusieurs arguments et qui s'applique notamment aux têtes lexicales.

40. Cela est vrai également de la relation entre le sujet de phrase et le prédicat verbal puisque le rôle thématique du sujet est assigné par le verbe de manière compositionnelle (verbe et complément(s)). Il n’y a donc pas de dépendance thématique directe entre le verbe et le sujet.
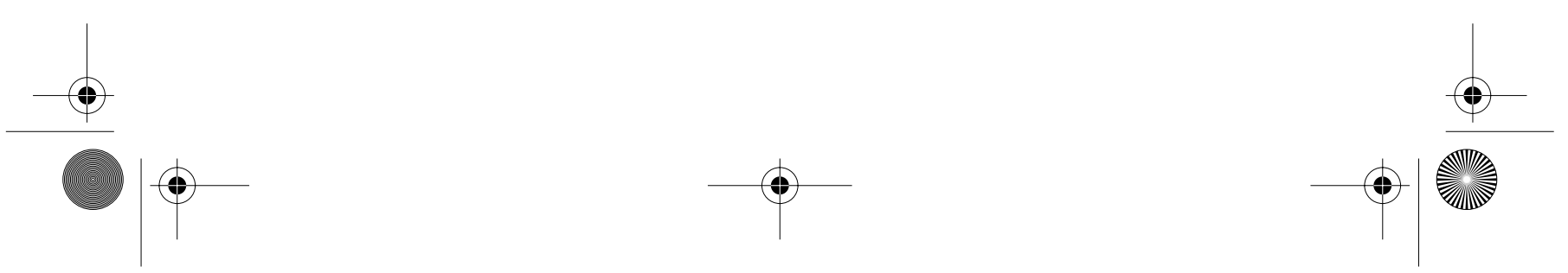
locatif. (41c) et (42c) explicitent les deux structures correspondantes en ne retenant du cas locatif que le trait pertinent de la relation.

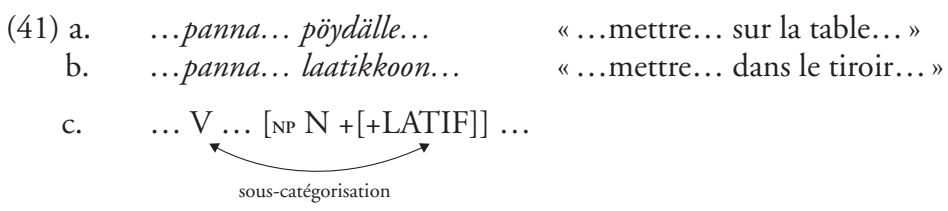
(42) a. ... kirjat... pöydälle... "... les livres... sur la table...»
b. ... kirjat... laatikkoon... "... les livres... dans le tiroir..."
c. $\quad \ldots[\mathrm{NP}] \ldots[\mathrm{NP} \mathrm{N}+[ \pm$ INTERNE $]] \ldots$

Étant donné les spécificités du système des cas locatifs du finnois, la relation spatiale de type porteur/porté $e^{41}$ (cas particulier d'incohérence) implique le cas allatif ([-INTERNE]) en (42a) et ce trait est associé à l'entité / fonction [LIEU ON ([CHOSE ] )], alors que la relation contenant / contenu ${ }^{42}$ (cas particulier de cohérence) justifie le cas illatif en (42b) et l'entité/fonction [LIEU IN ([CHOSE ] )]. Dans la relation de prédication entre un locatif et son sujet de prédication, la valeur du trait $[ \pm$ INTERNE] n'est pas déterminée uniquement par les propriétés ontologiques du site mais également par le choix lexical du sujet de prédication (43a-b) et par la nature de la relation entre les deux (43c).

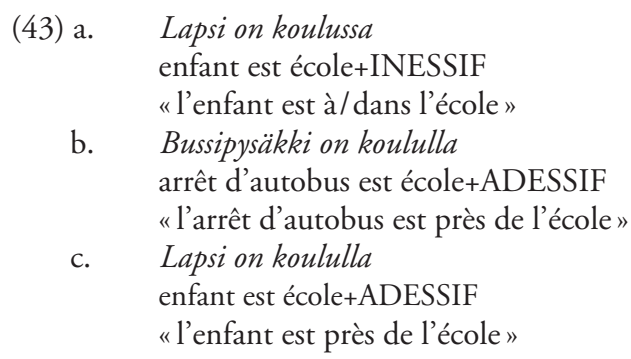

Considérons maintenant l'aspect syntaxique de la relation de prédication définie par les compléments locatifs. L'analyse proposée ici met en rapport les traits morphologiques des suffixes de cas avec les entités / fonctions de la structure conceptuelle de Jackendoff, sans aucune

41. Vandeloise 1986.

42. Ibid. 
indication sur la structure syntaxique des phrases avec locatif ni sur la configuration de la prédication syntaxique. J'adopterai ici les hypothèses majeures du programme minimaliste de la grammaire générative ${ }^{43}$ en considérant que les traits morphologiques des suffixes de cas constituent des têtes syntaxiques dont la position relative est définie par les relations de dépendances immédiates. Les traits d'orientation sont en relation locale avec le verbe sous-catégorisé pour ces traits; la projection maximale des traits d'orientation est soit complément du verbe soit adjointe au verbe $\left.{ }^{44}\right)$. Le trait de localisation $[ \pm$ INTERNE] définit une relation locale de sélection avec une expression référentielle spécifiée pour le nombre. Le système des cas locatifs du finnois impose également qu'un trait d'orientation sélectionne une projection du trait de localisation lorsque le site est une expression référentielle (ce qui n'est pas le cas avec les noms non référentiels qui prennent un des trois cas non locatifs (essif, translatif et partitif ${ }^{45}$ ). Cette contrainte transposé dans les termes de la structure conceptuelle signifie qu'une entité [CHEMIN ] doit obligatoirement comporter une entité complexe [LIEU ON/IN ([CHOSE ])] si celle-ci contient une expression référentielle. Cette relation de dépendance syntaxique entre les deux dimensions du système casuel finnois doit être spécifiée :

(44) Les traits d'orientation des cas locatifs $[ \pm \mathrm{ESSSIF}, \pm$ ILLATIF] sélectionnent le trait de localisation $[ \pm$ INTERNE] si, et seulement si, l'entité [LIEU ] contient une expression référentielle.

La représentation partielle (45) est conforme à l'organisation hiérarchisée des projections fonctionnelles proposée par Cinque ${ }^{46}$ pour les prépositions de l'anglais, du néerlandais ${ }^{47}$ et des cas locatifs du lesghien (langue caucasique du Nord-Est) ${ }^{48}$. Pour les cas locatifs du finnois, cette structure est également en accord - après déplacement des têtes - avec l'analyse diachronique de Hakulinen ${ }^{49}$ :

43. Chomsky 1995.

44. Je laisse de côté l'analyse des verbes transitifs à complément locatif comme des prédicats complexes contenant plusieurs projections verbales (Larson 1988, Hale et Keyser 1993, Radford 1997).

45. Voir la note 37

46. Cinque 1999.

47. Van Riemsdijk 1978 1990, Koopman 1993.

48. Haspelmath 1993, Van Riemsdijk 1996.

49. Hakulinen 1968.

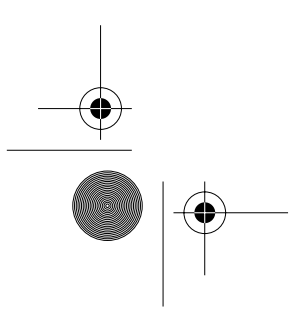


(45)

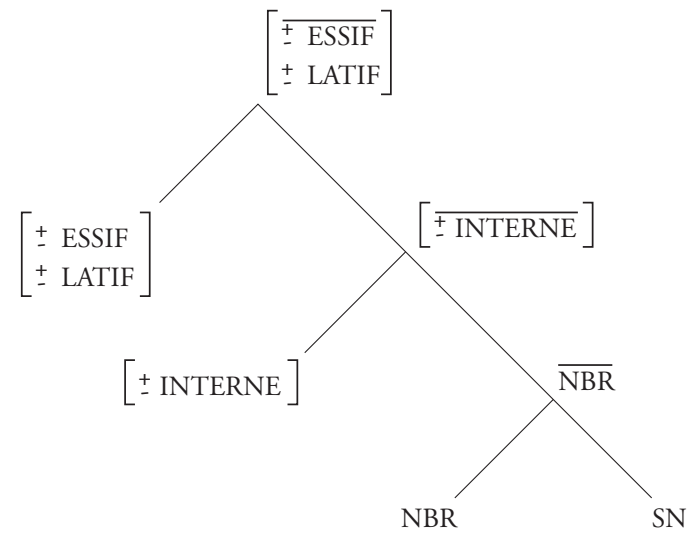

Après déplacement des têtes ${ }^{50}$, la structure locative (45) permet de former le mot fléchi kaduilla "dans les rues». Les suffixes *l et *nA, empruntés à l'analyse diachronique, représentent respectivement l'épel des traits de localisation et d'orientation ${ }^{51}$ :

(46) kaduilla "dans les rues"

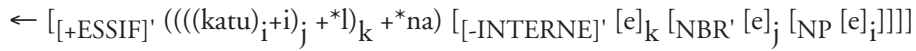

Selon Rothstein ${ }^{52}$, qui a initié les travaux sur la prédication syntaxique, les projections maximales se répartissent en arguments et prédicats dont la distribution est réglée par deux principes indépendants de bonne formation des phrases; le critère thématique (un argument doit entrer dans une relation de dépendance thématique définie par une tête lexicale) et le principe de prédication qui stipule que tout prédicat doit être en relation avec un sujet (de prédication) ${ }^{53}$. Ce principe s'applique aussi bien aux prédicats primaires (prédicats définis par la présence d'un morphème de temps) qu'aux prédicats seconds (prédicats ayant pour sujet de prédication un constituant qui ne dépende pas des propriétés thématiques du prédicat) ${ }^{54}$. Pour illustrer ce dernier

50. Travis 1984, Radford 1997.

51. Voir la note 26.

52. Rothstein 1983, 1995.

53. Plus précisément (Rothstein 1995) : «Tout prédicat syntaxique doit être syntaxiquement saturé. "

54. Rothstein 1983 (définition légèrement modifiée) : 1) «X est un prédicat primaire de $\mathrm{Y}$ si, et seulement si, $\mathrm{X}$ et $\mathrm{Y}$ forment un constituant -marqué ou contenant une tête [+TPS]. 2) X est un prédicat second de $Y$ si, et seulement si $Y$ est un $S N$-marqué par une tête lexicale autre que X.
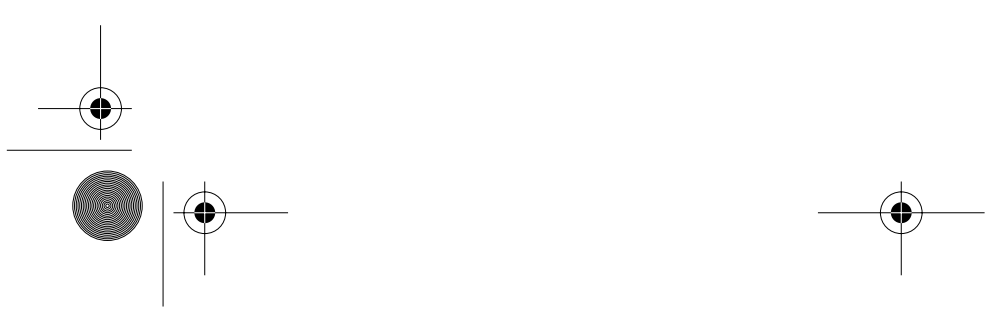
principe, considérons les phrases suivantes dans lesquelles le prédicat locatif et son sujet de prédication sont soulignés :

a. Suvi meni asemalle

Suvi est allée gare+ALLATIF

"Suvi est allée à la gare»

b. Suvi pani kirjat pöydülle

Suvi a mis les livres table+ALLATIF

"Suvi a mis/posé les livres sur la table»

c. Suvi luki sanomalehdet kirjastossa

Suvi a lu les journaux bibliothèque +INESSIF

"Suvi a lu les journaux dans la bibliothèque"

d. Suvi näki auton kadulla

Suvi a vuvoiture rue+ADESSIF

"Suvi a vu la voiture dans la rue»

e. Suvi tuli kädet askussa

Suvi est venue les mains poche+INESSIF

"Suvi est venue les mains dans les poches"

Dans ces différentes structures, le locatif est soit un complément du verbe (47a-b), soit un circonstant (47c-d), soit un prédicat inclus dans une construction absolue (47c). Quel que soit le point d'ancrage du locatif dans les représentations syntaxiques, la valeur du trait de localisation $[ \pm I N T E R N E]$ est déterminée par la relation spatiale entre le site (le prédicat locatif) et la cible (son sujet de prédication). L'analyse de la prédication syntaxique de Rothstein implique une contrainte d'ordre structurale qui repose sur la relation de m-commande ${ }^{55}$ :

(48) a. Toute projection maximale non marquée Y doit être reliée en S-structure à un argument $\mathrm{X}$ tel que $\mathrm{X} \mathrm{m}$-commande $\mathrm{Y}$ et $\mathrm{Y} \mathrm{m}$-commande $\mathrm{X}$.

b. A m-commande $\mathrm{B}$, si la première projection maximale qui domine $\mathrm{A}$ domine $\mathrm{B}$.

Le point important de cette contrainte est que la relation structurale entre un prédicat et son sujet est donnée pour symétrique ${ }^{56}$. Par manque de place, je n'entrerai pas dans le détail des configurations syntaxiques des phrases (47a-e). Deux remarques seulement: 1) Pour le moins, le sujet de prédication des phrases (47a-e) précède et m-commande le prédicat locatif dans tous les exemples, 2) la relation de mcommande réciproque est mise en difficulté par les phrases (47a) et (47e) dans lesquelles le statut du locatif est moins clair vis à vis de la distinction

55. Aoun et Sportiche 1983.

56. Voir à ce propos les différentes analyses présentées et commentées dans Wincker 1997. 
entre prédicat principal et prédicat second. Cela concerne les phrases (47a) et (47e) : le sujet de prédication de (47a) se confond avec le sujet de phrase, alors que le locatif forme avec le verbe un prédicat complexe (47a), et le sujet de prédication est inclus en (47e) dans une proposition indépendante qui fonctionne comme circonstant de manière.

En conclusion, je propose l'hypothèse suivante pour expliquer la seconde dimension du système des cas locatifs du finnois :

(49) Le trait de localisation des cas locatifs ([ \pm INTERNE]) introduit et identifie une relation de prédication syntaxique entre deux termes dont le prédicat locatif représente le site et le sujet de prédication, la cible.

Une remarque d'ordre typologique pour terminer. L'analyse que j'ai présentée des cas locatifs du finnois a pour effet d'assigner à l'un des traits morphologiques des suffixes locatifs - le trait de localisation - un statut de tête fonctionnelle assurant le rôle de médiateur/marqueur d'une relation prédicative. Les exemples (50) et (51), empruntés à des langues différentes, montrent que cette même fonction syntaxique peut être assurée par d'autres catégories fonctionnelles (nombre, genre, cas). Dans les exemples (50a-b), extraits d'une grammaire de l'avar ${ }^{57}$, le locatif s'accorde en nombre et en genre avec son sujet de prédication (sujet de la phrase):

\section{(50) a. was roq'ów xxút'un wígo}

jeune homme maison+INESSIF+SG/M resté est

«le jeune homme est resté dans la maison»

b. yas roq'óy xxút'un yúgo jeune fille maison+INESSIF+SG/F resté est

"la jeune fille est resté dans la maison »

Dans cet autre exemple, emprunté à Hale ${ }^{58}$, le locatif Walbiri (langue aborigène d'Australie) s'accorde en cas avec le sujet (reprise du suffixe d'ergatif sous une forme différente) :

(51) Ngarrkangku opalangu yankirijarra luwarnu ngapangkarlu homme+ERG AUX deux émeus abattu point d'eau+LOC+ERG

L'homme a abattu les deux émeus au point d'eau»

57. Charachidzé 1981.

58. Hale 1981. 


\section{Références bibliographiques}

Aoun J. et Sportiche D. (1983), «On the Formal Theory of government ", Linguistic Review, 2, p. 211-236.

Blake B. J. (1994), Case, Cambridge, Cambridge University Press.

Charachidzé G. (1981), Grammaire de la langue avar, Paris, Éditions Jean Favard.

Сномsкy N. (1986), Knowledge of Language: Its Nature, Origin, and Use, New York, Praeger.

Сномsкy N. (1995), The Minimalist Program, Cambridge, Mass., The MIT Press.

Cinque G. (1999), Adverbs and Functional Heads: a Cross-Linguistic Perspective, Oxford, Oxford University Press.

François J. (1996), «Reference to space and time through local adpositions and local cases: beyond the proposals of C. de Groot, J.H. Connolly and J.L. Mackenzie», Working Papers in Functional Grammar, $\mathrm{n}^{\circ} 63$.

François J. (2002), "Cognition spatiale et typologie des langues", Cabiers de la MRSH, n ${ }^{\circ}$ 30, p. 51-64.

HaLe K. (1981), On the position of Walbiri in a Typology of the Base, Bloomington, Indiana University Linguistics Club.

Hale K. and Keyser S.J. (1993), «On argument structure and the lexical expression of semantic relations", in The view from Building 20, K. Hale et S.J. Keyser (éd.), Cambridge, Mass., The MIT Press.

Halliday (1967), "Notes on Transitivity and Theme in English", Part 1, Journal of linguistics 3, p. 37-81.

Hakulinen L. (1968), Suomen kielen rakenne ja kehitus, $3^{\mathrm{e}}$ édition, Helsinki, Otava.

Haspelmath M. (1993), A Grammar of Lezgian, Berlin, Mouton de Gruyter.

HJelmslev L. (1935), "La catégorie du cas I ", Acta Jutlandica, VII.

HJelmslev L. (1937), "La catégorie du cas II », Acta Jutlandica, IX.

Iкоца O. (1983), Nykysuomen käsikirja, Espoo, Weilin - Göös.

Jackendoff R. (1983), Semantics and Cognition, Cambridge, Mass., The MIT Press.

Jackendoff R. (1990), Semantic Structures, Cambridge, Mass., The MIT Press. 
Jönsson-Korhola H. et White L. (1997), Tarkista tästä, Helsinki, Finn Lectura.

Kendall M.B. (1975), "The /-k/, /-m/problem in Yavapai syntax", International Journal of American Linguistics, 41-1, p. 1-9.

Karlsson F. (1999), Finnish : an Essential Grammar, Londres-New York, Routledge.

Koopman H. (1993), "The structure of Dutch Pps», article non publié, UCLA.

LARSON R. (1988), "On the double objet construction", Linguistic Inquiry 19, p. 335-391.

LeINo P. et al., (1990), Suomen kielen paikallissija konseptualisessa smantiikassa, Kieli 5, Helsingin Yliopiston Suomen Kielen Laitos.

Mel'Čuk I. (1994), Cours de morphologie générale, volume 2 : significations morphologiques, Montréal - Paris, Les Presses universitaires de Montréal - CNRS Éditions.

Napoli D.J. (1989), Predication Theory: a case study for indexing theory, Cambridge, Mass., Cambridge University Press.

Nikanne U. (1989), "On locative Cases in Finnish", Papers from the $11^{\text {th }}$ Scandinavian Conference of Linguistics, J. Niemi (éd.), University of Joensuu.

RAdFord A. (1997), Syntax, a minimalist introduction, Cambridge Mass., Cambridge University Press.

Remes H. (1982), Viron kielioppi, Porvoo, WSOY.

Riemsdijk H. van (1978), A case study in Syntactic Markedness, Lisse, The Peter de Ridder Press.

Riemsdijk H. van (1990), "Functional Prepositions» in Unity in Diversity, Harm Pinkster et I. Genée (éd.), Dordrecht, Foris, p. 229-241.

Riemsdijk H. van (1996), "Categorial Feature Magnetism: the Extension and Distribution of Projections ", article non publié, Tilburg University.

Rothstein S.D. (1983), The Syntactic Form of Predication, Ph.D. dissertation, MIT.

Simpson J. (1981), "Finnish Essive and Translative», article non publié.

Stowell T.A., (1981), The Origins of Phrase Structure, Ph.D. dissertation, MIT.

Vandeloise Cl. (1986), L'Espace en français, Paris, Seuil. 
VANDELOISE Cl. (1987), «La préposition à et le principe d'anticipation ", Langue française, 77, p. 77-111.

VANDELoise Cl. (1988), «Les usages spatiaux statiques de la préposition à», Cahier de lexicologie, 53, p. 119-148.

Williams E. (1981), «Predication», Linguistic Inquiry 11-1, p. 203238.

Winkler S. (1997), Focus and Secondary Predication, Studies in Generative Grammar 43, Berlin - New York, Mouton de Gruyter.
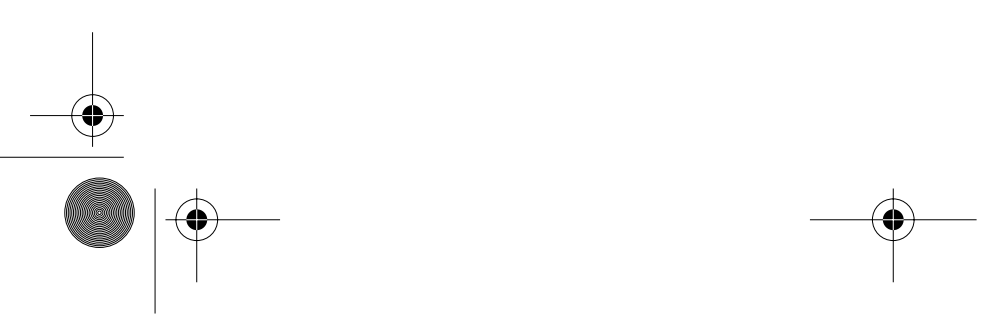